The author has the right to post and update the article on a free-access e-print server using files prepared and formatted by the author. Any such posting made or updated after acceptance of the article for publication by APS should include a link to the online APS journal article abstract. In all cases, the appropriate bibliographic citation and notice of the APS copyright must be included.

PHYSICAL REVIEW B

VOLUME 57, NUMBER 2

1 JANUARY 1998-II

\title{
Magnetoconductance due to variable-range hopping in quasi-two-dimensional systems: Application to $\mathrm{PrBa}_{2} \mathrm{Cu}_{3} \mathrm{O}_{7-\delta}$
}

\author{
R. B. Thompson and M. Singh \\ Department of Physics and Astronomy, University of Western Ontario, London, Ontario, Canada N6A $3 K 7$
}

(Received 14 May 1997; revised manuscript received 8 August 1997)

\begin{abstract}
In this paper, we have developed a theory of magnetoconductance (magnetoresistance) due to variable-range hopping for quasi-two-dimensional systems. We have included the effect of electric fields on the calculation of the magnetoconductance. The effects of scattering and electron-electron interactions have also been included in our theory. We found analytical expressions for the conductivity for both the scattering and nonscattering cases, and obtained electric- and magnetic-field-dependent power laws in certain approximations. We found that the electric and magnetic-field dependences of the magnetoconductance had different power laws for the scattering and nonscattering cases. We tried to explain the van Ancum et al. magnetoconductance experiments of $\mathrm{PrBa}_{2} \mathrm{Cu}_{3} \mathrm{O}_{7-\delta}$ (PBCO) thin films by using our theory. A good agreement between theory and experiment was found if we included the effect of scattering. In the above PBCO films, it was found that the approximate value of the concentration of localized states lies between $10^{11}$ and $10^{12} \mathrm{~cm}^{-2}$. [S0163-1829(98)00602-X]
\end{abstract}

DOI: https://doi.org/10.1103/PhysRevB.57.1284

\section{INTRODUCTION}

There has been a considerable interest in the study of hopping conduction in low-dimensional systems such as oxide superconductors and related materials. ${ }^{1,2}$ A material that figures highly in much of this research is $\mathrm{PrBa}_{2} \mathrm{Cu}_{3} \mathrm{O}_{7-\delta}$ (PBCO) because of its current and potential uses in the technology of high-temperature superconducting junctions. PBCO has been shown to conduct via a variable-range hopping (VRH) mechanism along its $\mathrm{CuO}_{2}$ planes ${ }^{1,3}$ and so quasi-two-dimensional (QTD) theories are required to explain its properties. Recently, we have developed a theory for variable-range-hopping conductivity in the presence of electric fields for QTD and quasi-one-dimensional systems., We have also included the effect of electron-electron interactions in our theoretical calculations. We applied our theory to explain the electric-field-dependent conductivity data of Kabasawa et $a l .{ }^{1}$ for PBCO-based S/N/S junctions and found a good agreement between theory and experiment.

Recently van Ancum et al. ${ }^{5}$ have measured the magneticfield-dependent conductivity in PBCO thin films and suggested that the magnetoconductance in these films is due to variable-range hopping. They modified three-dimensional expressions of the magnetoconductance ${ }^{6}$ for the twodimensional case and tried to fit their data by using these expressions. They did not include in their expressions how the hopping exponents depended on the material parameters, which allowed them great latitude in fitting the experimental data. The effect of a magnetic field on VRH conduction in three-dimensional systems has been examined using the percolation method ${ }^{6}$ for strong (i.e., $\lambda \ll a_{0}$ ) magnetic fields and weak (i.e., $\lambda \gg a_{0}$ ) magnetic fields, where $\lambda=\sqrt{\hbar / q H}, a_{0}$ is the localization length in the absence of a magnetic field, $q$ is the charge of a carrier, and $H$ is the magnetic field.

In this paper, we have derived the expressions for the magnetoconductance for QTD systems for weak and strong magnetic fields by using the method developed by us. ${ }^{3,4}$ This method allows for the inclusion of all pre-exponential fac- tors, and the inclusion of all relevant material parameters in the exponents. This approach differs significantly from the percolation method in the calculation of mobility and conductivity, and is better suited for obtaining analytical results for cases where the electric field is to be included. We have derived formulas for the magnetoconductance both with and without the inclusion of an electric field. In the former case, the magnetic-field dependency has been calculated to a higher order than in any previous work. In the latter case, unified formulas for the conductivity including all temperature, electric field, and magnetic-field dependences have been presented. The effects of scattering and electronelectron interactions have also been included in our theory. The effect of scattering was investigated by Shklovskii in QTD systems by using the percolation method. ${ }^{6}$ Our expression of magnetoconductance in the presence of scattering can easily be reduced to that of Shklovskii by making appropriate approximations.

We found that the logarithm of our expression of the magnetoconductance for the constant DOS in certain approximations is proportional to $H^{1 / 2}$ for strong magnetic fields without scattering. This is in general agreement with the expressions given in Refs. 5 and 7. For electron-electron interactions, the result is $H^{1 / 3}$. For weak magnetic fields without scattering we found that the logarithm of the constant density of states (DOS) magnetoconductivity expression gave an $H^{2}$ magnetic-field dependence, consistent with the expression of van Ancum. ${ }^{5}$ For electron-electron interactions the result is also an $H^{2}$ dependence.

We used our theory to explain the PBCO thin-film magnetoconductance experiments of van Ancum et al. It is found that these experiments cannot be explained by using the expressions of magnetoconductance in the absence of scattering. When the effect of scattering is included in the calculations, a good agreement between theory and experiments is found. One fitting parameter is used to get a good agreement between theory and experiments. From our theoretical calculations, we found that the concentration of localized states 
lies between $10^{11}\left(1 / \mathrm{cm}^{2}\right)$ and $10^{12}\left(1 / \mathrm{cm}^{2}\right)$. Experimentally, the effect of scattering has also been observed in threedimensional systems. ${ }^{8}$ If we include the effects of magnetic fields and scattering, we find for the PBCO thin films of van Ancum that the logarithm of the conductivity varies as $\beta^{-1 / 2} H^{4 / 3}$. Here, $\beta$ is proportional to the electric field $E, \beta$ $=q E / 2 \alpha k_{B} T$ with $\alpha=1 / a_{0}$. This shows that the effects of scattering on a QTD system can be observed through the electric and magnetic-field behavior of the conductivity.

\section{HOPPING MAGNETOCONDUCTANCE}

In this section, we will calculate analytical expressions of conductivity in the presence of weak and strong magnetic fields.

\section{A. Weak magnetic fields}

Following our previous work, ${ }^{4}$ we assume the localized states are randomly distributed in energy and twodimensional space coordinates (hopping space) and form a discrete array of sites. We assume the spread in energies of the states is fairly small and near the Fermi level. This allows us to ignore the effects of correlations. ${ }^{9}$ A magnetic field is applied transverse to the two-dimensional space coordinates. For QTD systems, the probability of a charge carrier hopping from an initial state, $E_{i}$ to a final state, $E_{j}$ in the hopping space for weak magnetic fields $\left(\lambda \gg a_{0}\right.$ while $a_{0} \leqslant \rho_{i j}$ $\left.\leqslant \lambda^{2} / a_{0}\right)$ is given by

$$
\begin{gathered}
W(R)=W_{0} \exp (-R), \\
R=x+\gamma x^{3}+\omega-\varepsilon, \quad \omega>\varepsilon, \\
R=x+\gamma x^{3}, \quad \omega<\varepsilon,
\end{gathered}
$$

where $W_{0}$ is a constant and $R$ is the distance between two states in the energy-space coordinates, called the range. We obtained the expression of $R$ by considering the asymptotic magnetic field wave functions of Shklovskii. ${ }^{6}$ Here, $\gamma$ $=a_{0}^{4} q^{2} H^{2} / 96 \hbar^{2}, x=2 \rho_{i j} / a_{0}, \omega=E_{j} / k_{B} T$, and $\varepsilon=E_{i} / k_{B} T$. Also, $a_{0}$ is the localization length, and $\rho_{i j}$ is the distance between two sites $i$ and $j$ in the two-dimensional space coordinates. Note that the first term in $R$ is the dominant term compared to the subsequent terms. To evaluate the analytical results in the weak magnetic-field case, we considered this condition. The effect of a magnetic field is entirely contained in $\gamma$.

In the evaluation of the magnetoconductance, we have considered the energy-independent and energy-dependent densities of states (DOS) for the localized electrons. Mott and others ${ }^{10,11}$ assumed that the DOS of localized electrons is constant and does not depend on energy. Later, Efros and Shklovskii ${ }^{6}$ showed that if one includes the effect of electron-electron interactions, the DOS is not constant but depends on the energies of the localized electrons. They found that

$$
\begin{gathered}
D(\omega)=D_{0} \quad \omega>\Delta_{c g} / k_{B} T, \\
D(\omega)=D_{1} k_{B} T \omega^{(d-1)} \quad \omega<\Delta_{c g} / k_{B} T,
\end{gathered}
$$

where $\Delta_{c g}$ is called the Coulomb gap, $d$ is the dimensionality of the system, and $D_{0}$ and $D_{1}$ are constants. Here, energy is measured with respect to the Fermi energy. In the rest of the paper, we will attach subscripts $c$ and $e$ to the physical quantities related to the constant DOS and DOS with electronelectron interactions, respectively.

The following analytical expressions for the critical hopping distance $R_{n n}$ are obtained by using Eqs. (2.1), (2.2), and the method of Singh and Thompson ${ }^{3,4}$ to first order in $\varepsilon$ and second order in $\gamma$ :

$$
\begin{aligned}
& R_{n n}^{c}=R^{c}\left(1-\frac{\varepsilon}{R^{c}}+\frac{56 \varepsilon \gamma^{2}\left(R^{c}\right)^{3}}{25}+\frac{2 \gamma\left(R^{c}\right)^{2}}{5}-\frac{9 \gamma^{2}\left(R^{c}\right)^{4}}{25}\right), \\
& R_{n n}^{e}=R^{e}\left(1-\frac{\varepsilon}{R^{e}}+\frac{9 \varepsilon \gamma^{2}\left(R^{e}\right)^{3}}{10}+\frac{\gamma\left(R^{e}\right)^{2}}{5}-\frac{39 \gamma^{2}\left(R^{e}\right)^{4}}{200}\right),
\end{aligned}
$$

where $R^{c}=\left(T / T_{c}\right)^{-1 / 3}, R^{e}=\left(T / T_{e}\right)^{-1 / 2}, T_{c}=12 \alpha^{2} / k_{B} D_{0} \pi$, and $T_{e}=\sqrt{48 \alpha^{2} / k_{B} D_{1} \pi}$.

Similarly, using Eq. (2.3) and the method of Singh and Thompson, ${ }^{3,4}$ the following analytical expressions for the magnetoconductivity are obtained.

$$
\begin{gathered}
\sigma_{c}(H)=\frac{q^{2} \nu_{p} D_{0}}{8 \alpha^{2}}\left[\frac{2}{3}\left(\frac{T_{c}}{T}\right)^{1 / 3}-\frac{7}{5} \gamma\left(\frac{T_{c}}{T}\right)\right] \frac{\exp \left(-R_{0}^{c}\right)}{R_{1}^{c}} \\
\sigma_{e}(H)=\frac{q^{2} \nu_{p} D_{1} k_{B} T}{8 \alpha^{2}}\left[\frac{1}{2}\left(\frac{T_{e}}{T}\right)^{1 / 2}-\frac{3}{4} \gamma\left(\frac{T_{e}}{T}\right)^{3 / 2}\right] \frac{\exp \left(-R_{0}^{e}\right)}{\left(R_{1}^{e}\right)^{2}} .
\end{gathered}
$$

$R_{0}$ and $R_{1}$ are given as

$$
\begin{gathered}
R_{0}^{c}=\left(\frac{T_{c}}{T}\right)^{1 / 3}+\frac{2}{5} \gamma\left(\frac{T_{c}}{T}\right)-\frac{9}{25} \gamma^{2}\left(\frac{T_{c}}{T}\right)^{5 / 3} \\
R_{1}^{c}=1-\frac{56}{25} \gamma^{2}\left(\frac{T_{c}}{T}\right)^{4 / 3} \\
R_{0}^{e}=\left(\frac{T_{e}}{T}\right)^{1 / 2}+\frac{\gamma}{5}\left(\frac{T_{e}}{T}\right)^{3 / 2}-\frac{39}{200} \gamma^{2}\left(\frac{T_{e}}{T}\right)^{5 / 2} \\
R_{1}^{e}=1-\frac{9}{10} \gamma^{2}\left(\frac{T_{e}}{T}\right)^{2}
\end{gathered}
$$

to second order in $\gamma$. Note that if we consider only the magnetic-field dependence, then to first order in $\gamma$ we get that the logarithm of the conductivity is proportional to $H^{2}$ for the constant density of states, in agreement with the van Ancum percolation result. ${ }^{5}$ For electron-electron interactions, we get the new result that the logarithm of the magnetoconductance is proportional to $H^{2}$ also. When the magnetic field becomes zero, we see that we regain from Eq. (2.4) the wellknown Mott and Efros-Shklovskii hopping expressions for two-dimensional systems. 


\section{B. Strong magnetic fields}

In the presence of strong magnetic fields $\left(\lambda \ll a_{0}\right.$ while $\left.\rho_{i j} \gg \lambda^{2} / a_{0}\right)$, we obtain the following expression of the range using the asymptotic wave function of Shklovskii: ${ }^{6}$

$$
\begin{gathered}
R=\gamma x^{2}+\omega-\varepsilon, \quad \omega>\varepsilon \\
R=\gamma x^{2}, \quad \omega<\varepsilon
\end{gathered}
$$

for QTD systems, where $\gamma=\left(a_{0}^{2} / 8 \lambda^{2}\right)$. Using Eq. (2.6) and the method of Singh and Thompson, ${ }^{3,4}$ we get the expressions for $R_{n n}$ to first order in $\varepsilon$ as

$$
\begin{aligned}
& R_{n n}^{c}=R^{c}\left(1-\frac{\varepsilon}{R^{c}}\right), \\
& R_{n n}^{e}=R^{e}\left(1-\frac{\varepsilon}{R^{e}}\right),
\end{aligned}
$$

where $R^{c}=\left(T / T_{c}\right)^{-1 / 2}(2 \gamma / 3)^{1 / 2}$ and $R^{e}=\left(T / T_{e}\right)^{-2 / 3}(\gamma / 2)^{1 / 3}$. Following the method of Singh and Thompson, ${ }^{3,4}$ we get expressions of the magnetoconductivities for the constant and energy-dependent DOS, respectively, as

$$
\begin{gathered}
\sigma_{c}(H)=\frac{q^{2} \nu_{p} D_{0}}{16 \alpha^{2} \gamma} \exp \left[-\left(\frac{T}{T_{c}}\right)^{-1 / 2}\left(\frac{2 \gamma}{3}\right)^{1 / 2}\right], \\
\sigma_{e}(H)=\frac{q^{2} \nu_{p} k_{B} T D_{1}}{16 \alpha^{2} \gamma} \exp \left[-\left(\frac{T}{T_{e}}\right)^{-2 / 3}\left(\frac{\gamma}{2}\right)^{1 / 3}\right] .
\end{gathered}
$$

Note that the logarithm of the conductivity is proportional to $H^{1 / 2}$ for the case of the constant DOS, in agreement with Refs. 5 and 7 for strong magnetic fields. For electronelectron interactions, the logarithm is proportional to $H^{1 / 3}$.

\section{THE EFFECT OF ELECTRIC FIELDS ON MAGNETOCONDUCTANCE}

In this section, we will study the effect of electric fields on the magnetoconductance.

\section{A. Weak magnetic fields}

In the presence of an electric field, the expression of the range given in Eq. (2.1) for a weak magnetic field $\left(\lambda \gg a_{0}\right.$ while $a_{0} \leqslant \rho_{i j} \leqslant \lambda^{2} / a_{0}$ ) becomes

$$
\begin{gathered}
R=x(1+\beta \cos \theta)+\gamma x^{3}+\omega-\varepsilon, \quad \omega+x \beta \cos \theta>\varepsilon \\
R=x+\gamma x^{3}, \quad \omega+x \beta \cos \theta<\varepsilon .
\end{gathered}
$$

where $\beta$ is proportional to the electric field $E$, defined as $\beta$ $=q E / 2 \alpha k_{B} T$, and $q$ is the charge of one charge carrier. Using Eq. (3.1) and the method of Singh and Thompson,, ${ }^{3,4}$ we get the values of $R_{n n}$ as

$$
\begin{aligned}
R_{n n}^{c}= & R^{c}\left(1-\frac{c_{2}^{c}}{c_{1}^{c}} \frac{\varepsilon}{R^{c}}+\frac{2}{5} \frac{c_{3}^{c}}{c_{1}^{c}} \gamma R^{c 2}+2 \frac{c_{4}^{c}}{c_{1}^{c}} \gamma \varepsilon R^{c}\right. \\
& \left.-\frac{2}{3} \frac{c_{2}^{c} c_{3}^{c}}{\left(c_{1}^{c}\right)^{2}} \gamma \varepsilon R^{c}\right),
\end{aligned}
$$

$$
\begin{aligned}
R_{n n}^{e}= & R^{e}\left(1-\frac{c_{2}^{e}}{c_{1}^{e}} \frac{\varepsilon}{R^{e}}+\frac{\gamma}{5} \frac{c_{3}^{e}}{c_{1}^{e}} R^{e 2}+\frac{6}{5} \frac{c_{4}^{e}}{c_{1}^{e}} \gamma \varepsilon R^{e}\right. \\
& \left.-\frac{6}{5} \frac{c_{2}^{e} c_{3}^{e}}{\left(c_{1}^{e}\right)^{2}} \gamma \varepsilon R^{e}\right)
\end{aligned}
$$

where $R^{c}=\left(T / T_{c}\right)^{-1 / 3}\left(c_{1}^{c}\right)^{-1 / 3}, R^{e}=\left(T / T_{e}\right)^{-1 / 2}\left(c_{1}^{e}\right)^{-1 / 4}$. Following the method of Singh and Thompson ${ }^{3,4}$ we get the conductivity expressions for the constant and energydependent DOS, respectively, as

$$
\begin{aligned}
\sigma_{c}= & \frac{q \nu_{p} D_{0} k_{B} T}{4 \alpha E}\left[\frac{c_{5}^{c} c_{9}^{c}}{c_{7}^{c}}+\gamma\left(\frac{c_{5}^{c} c_{10}^{c}}{c_{7}^{c}}-\frac{2 c_{6}^{c}\left(c_{9}^{c}\right)^{3}}{c_{7}^{c}}\right.\right. \\
& \left.\left.+\frac{c_{8}^{c} c_{5}^{c}\left(c_{9}^{c}\right)^{3}}{\left(c_{7}^{c}\right)^{2}}\right)\right] \frac{\exp \left(-R_{0}^{c}\right)}{R_{1}^{c}}, \\
\sigma_{e}= & \frac{q \nu_{p} D_{1}\left(k_{B} T\right)^{2}}{4 \alpha E}\left[\frac{c_{5}^{e} c_{9}^{e}}{c_{7}^{e}}+\gamma\left(\frac{c_{5}^{e} c_{10}^{e}}{c_{7}^{e}}-\frac{2 c_{6}^{e}\left(c_{9}^{e}\right)^{3}}{c_{7}^{e}}\right.\right. \\
& \left.\left.+\frac{c_{8}^{e} c_{5}^{e}\left(c_{9}^{e}\right)^{3}}{\left(c_{7}^{e}\right)^{2}}\right)\right] \frac{\exp \left(-R_{0}^{e}\right)}{R_{1}^{e 2}}
\end{aligned}
$$

with

$$
\begin{aligned}
& R_{0}^{c}=c_{9}^{c}+\gamma c_{10}^{c}, \quad R_{1}^{c}=\left(\frac{c_{2}^{c}}{c_{1}^{c}}-\frac{2 c_{4}^{c}}{c_{1}^{c}} \gamma\left(c_{9}^{c}\right)^{2}+2 \frac{c_{2}^{c} c_{3}^{c}}{c_{1}^{c 2}} \gamma\left(c_{9}^{c}\right)^{2}\right), \\
& R_{0}^{e}=c_{9}^{e}+\gamma c_{10}^{e}, \quad R_{1}^{e}=\left(\frac{c_{2}^{e}}{c_{1}^{e}}-\frac{6 c_{4}^{e}}{5 c_{1}^{e}} \gamma\left(c_{9}^{e}\right)^{2}+\frac{6 c_{2}^{e} c_{3}^{e}}{5 c_{1}^{e 2}} \gamma\left(c_{9}^{e}\right)^{2}\right) .
\end{aligned}
$$

The parameters $c_{i}^{c}$ and $c_{i}^{e}$ appearing in the above expressions are presented in the Appendix. For $\beta \ll 1$ we get a $\beta^{2}$ dependence for the logarithm of the conductivity for both the constant DOS and the energy-dependent DOS. These results agree with the previous work. ${ }^{4}$ If we consider the magneticand electric-field dependence simultaneously, then for $\beta \gg 1$ we find that the logarithm of the conductivity is proportional to $\beta^{-1 / 3}+a_{1} H^{2} / \beta$ for the constant DOS and $\beta^{-1 / 2}$ $+a_{2} H^{2} / \beta^{3 / 2}$ for electron-electron interactions. This shows that the nature of the $\beta$ dependency of the conductivities will change upon increasing the magnetic field. For weak magnetic and electric fields $(\beta \ll 1)$ we get $\beta^{2}+a_{3} H^{2}$ $+a_{4} H^{2} \beta^{2}$ for both cases. The factors $a_{i}$ are numerical constants. For zero electric field, Eqs. (3.3) reduce to Eqs. (2.4). For zero magnetic field, we recover the expressions of our previous paper. $^{4}$

\section{B. Strong magnetic fields}

In the presence of an electric field, the expression of the range given in Eq. (2.6) for strong magnetic fields becomes 


$$
\begin{gathered}
R=\gamma x^{2}+x \beta \cos \theta+\omega-\varepsilon, \quad \omega+x \beta \cos \theta>\varepsilon \\
R=\gamma x^{2}, \quad \omega+x \beta \cos \theta<\varepsilon .
\end{gathered}
$$

Using Eq. (3.4) and the method of Singh and Thompson ${ }^{3,4}$ we get

$$
\begin{gathered}
R_{n n}^{c}=R^{c}\left(1-\frac{\varepsilon}{R^{c}}-\frac{\beta^{2}}{8 \gamma R^{c}}+\frac{\varepsilon \beta}{\pi \gamma^{1 / 2} R^{c 3 / 2}}\right), \\
R_{n n}^{e}=R^{e}\left(1-\frac{\varepsilon}{R^{e}}-\frac{\beta^{2}}{4 \gamma R^{e}}+\frac{\varepsilon \beta^{2}}{4 \gamma R^{e 2}}\right),
\end{gathered}
$$

where $R^{c}=\left(T_{c} / T\right)^{1 / 2}(2 \gamma / 3)^{1 / 2}, R^{e}=\left(T_{e} / T\right)^{2 / 3}(\gamma / 2)^{1 / 3}$, and $\gamma=a_{0}^{2} / 8 \lambda^{2}$. Using the method of Singh and Thompson ${ }^{3,4}$ we obtain the expressions for the conductivities for strong magnetic fields $\left(\lambda \ll a_{0}\right.$, while $\left.\rho_{i j} \gg \lambda^{2} / a_{0}\right)$ and weak electric fields $(\beta<1)$,

$$
\begin{gathered}
\sigma_{c}=\frac{q \nu_{p} D_{0} k_{B} T}{8 \alpha E}\left[\frac{\beta}{\gamma}-\frac{\beta^{2}}{\pi \gamma^{3 / 2}}\left(\frac{T}{T_{c}}\right)^{1 / 4}\left(\frac{3}{2 \gamma}\right)^{1 / 4}\right] \frac{\exp \left(-R_{0}^{c}\right)}{R_{1}^{c}} \\
\sigma_{e}=\frac{q \nu_{p} D_{1}\left(k_{B} T\right)^{2}}{8 \alpha E} \frac{\beta}{\gamma} \frac{\exp \left(-R_{0}^{e}\right)}{\left(R_{1}^{e}\right)^{2}}
\end{gathered}
$$

to second order in $\beta$, where

$$
\begin{gathered}
R_{0}^{c}=\left(\frac{T_{c}}{T}\right)^{1 / 2}\left(\frac{2 \gamma}{3}\right)^{1 / 2}-\frac{\beta^{2}}{8 \gamma}, \quad R_{1}^{c}=1-\frac{\beta}{\pi \gamma^{1 / 2}}\left(\frac{T}{T_{c}}\right)^{1 / 4}\left(\frac{3}{2 \gamma}\right)^{1 / 4}, \\
R_{0}^{e}=\left(\frac{T_{e}}{T}\right)^{2 / 3}\left(\frac{\gamma}{2}\right)^{1 / 3}-\frac{\beta^{2}}{4 \gamma}, \quad R_{1}^{e}=1-\frac{\beta^{2}}{4 \gamma}\left(\frac{T}{T_{e}}\right)^{2 / 3}\left(\frac{2}{\gamma}\right)^{1 / 3} .
\end{gathered}
$$

Note that the logarithm of the conductivity is proportional to $H^{1 / 2}$ and $\beta^{2}$ for the case of the constant density of states, and is proportional to $H^{1 / 3}$ and $\beta^{2}$ in the presence of electronelectron interactions. Considering the magnetic and electric fields together, the logarithms of the conductivities give $H^{1 / 2}-a_{5} \beta^{2} H^{-1}$ for the constant DOS and $H^{1 / 3}-a_{6} \beta^{2} H^{-1}$ for electron-electron interactions, where $a_{5}$ and $a_{6}$ are numerical constants. We see again that increasing magnetic fields will affect the electric-field behavior. For zero electric fields, Eq. (3.6) reduces to Eq. (2.8). We found that it was difficult to get an analytical expression for the conductivity for general electric fields.

\section{SCATTERING}

In this section we will include the effect of scattering on the conductivity in the presence of magnetic and electric fields. As the impurity concentrations in a sample increase, the average hopping length exceeds the mean distance between impurities. Thus a hopping electron meets many other impurities and scattering between the electron and impurities occur. It is evident from three-dimensional experiments that scattering plays an important role in the understanding of VRH magnetoconductance. ${ }^{8}$ In the presence of scattering, the wave function, $F(\rho)$ is written as ${ }^{6}$

$$
F(\rho)=\exp \left(-\frac{\rho}{b}\right)
$$

where the parameter $b$ is called the scattering length and is written as follows for two-dimensional systems: $:^{6}$

$$
\begin{gathered}
b=\frac{1}{\alpha}\left[1-\left(\frac{1}{\alpha \lambda}\right)^{4 / 3} L\right], \quad \lambda \gg N^{-1 / 2} \\
b=\frac{1}{\alpha}\left[1-q^{\prime}\left(\frac{1}{N^{1 / 2} \alpha \lambda^{2}}\right)^{4 / 3}\right], \quad\left(\alpha N^{1 / 2}\right)^{-1 / 2} \ll \lambda \ll N^{-1 / 2}, \\
b=s\left(\lambda^{2} N^{1 / 2}\right), \quad \lambda \ll\left(\alpha N^{1 / 2}\right)^{-1 / 2}
\end{gathered}
$$

where $N$ is the two-dimensional concentration of donor sites, $s$ and $q^{\prime}$ are numerical constants, and $L$ is a logarithmic factor. This logarithmic factor is related to the size of a scattering region and its physical interpretation is explained in detail in Ref. 12. Using Eq. (4.1), we obtained the following expression of $R$ :

$$
\begin{gathered}
R=x(1+\beta \cos \theta)+\omega-\varepsilon, \quad \varepsilon<\omega+x \beta \cos \theta, \\
R=x, \quad \varepsilon>\omega+x \beta \cos \theta,
\end{gathered}
$$

where now $x \equiv 2 \rho_{i j} / b$. Note that Eq. (4.5) includes the effects of scattering, magnetic, and electric fields. The calculated expressions of the conductivities in our previous article $^{4}$ would still be valid if we replace the localization length $a_{0}$ with the scattering length $b$. Therefore the expressions of the magnetoconductivities for the constant DOS and for electron-electron interactions can be written as

$$
\begin{aligned}
\sigma_{c}= & \frac{c_{0} T G_{c}(\beta) b}{E}\left(\frac{R_{c}^{0}+1}{R_{c}^{1}}\right) \exp \left[-\left(\frac{T}{T_{c}}\right)^{-1 / 3}\right. \\
& \left.\times\left(\frac{1}{2}+\frac{2 \beta}{\pi}+\eta_{1}\right)^{-1 / 3}\right], \\
\sigma_{e}= & \frac{c_{1} T^{2} G_{e}(\beta) b}{E}\left(\frac{R_{e}^{0}+2}{\left(R_{e}^{1}\right)^{2}}\right) \exp \left[-\left(\frac{T}{T_{e}}\right)^{-1 / 2}\right. \\
& \left.\times\left(\frac{1}{2}+\frac{2 \beta}{\pi}+\eta_{1}+\frac{3 \beta^{2}}{4}\right)^{-1 / 4}\right],
\end{aligned}
$$

respectively, where $R_{c}^{0}, R_{c}^{1}, R_{e}^{0}, R_{e}^{1}, G_{c}(\beta)$, and $G_{e}(\beta)$ can be found from our previous work. ${ }^{4}$ The parameters $c_{0}$ and $c_{1}$ are changed from before ${ }^{4}$ and are now $c_{0}=q k_{B} D_{0} \nu_{p} / 4$ and $c_{1}=q\left(k_{B}\right)^{2} D_{1} \nu_{p} / 4$. Likewise, $T_{c}$ and $T_{e}$ are changed, and now depend on the scattering length $b$. They will be given as $T_{c}=12[\Gamma(4 / 3)]^{3} / \pi D_{0} k_{B} b \quad$ and $T_{e}=\left\{48[\Gamma(5 / 4)]^{2 /}\right.$ $\left.\pi D_{1} k_{B}^{2} b^{2}\right\}^{1 / 2}$. Notice that $R_{c}^{0}$ and $R_{e}^{0}$ now depend on the magnetic field through their dependence on $T_{c}$ and $T_{e}$. The logarithms of the conductivities will behave as $b^{-1 / 3}$ for the constant DOS and $b^{-1 / 2}$ for electron-electron interactions, with respect to the scattering length. The exact form of the 


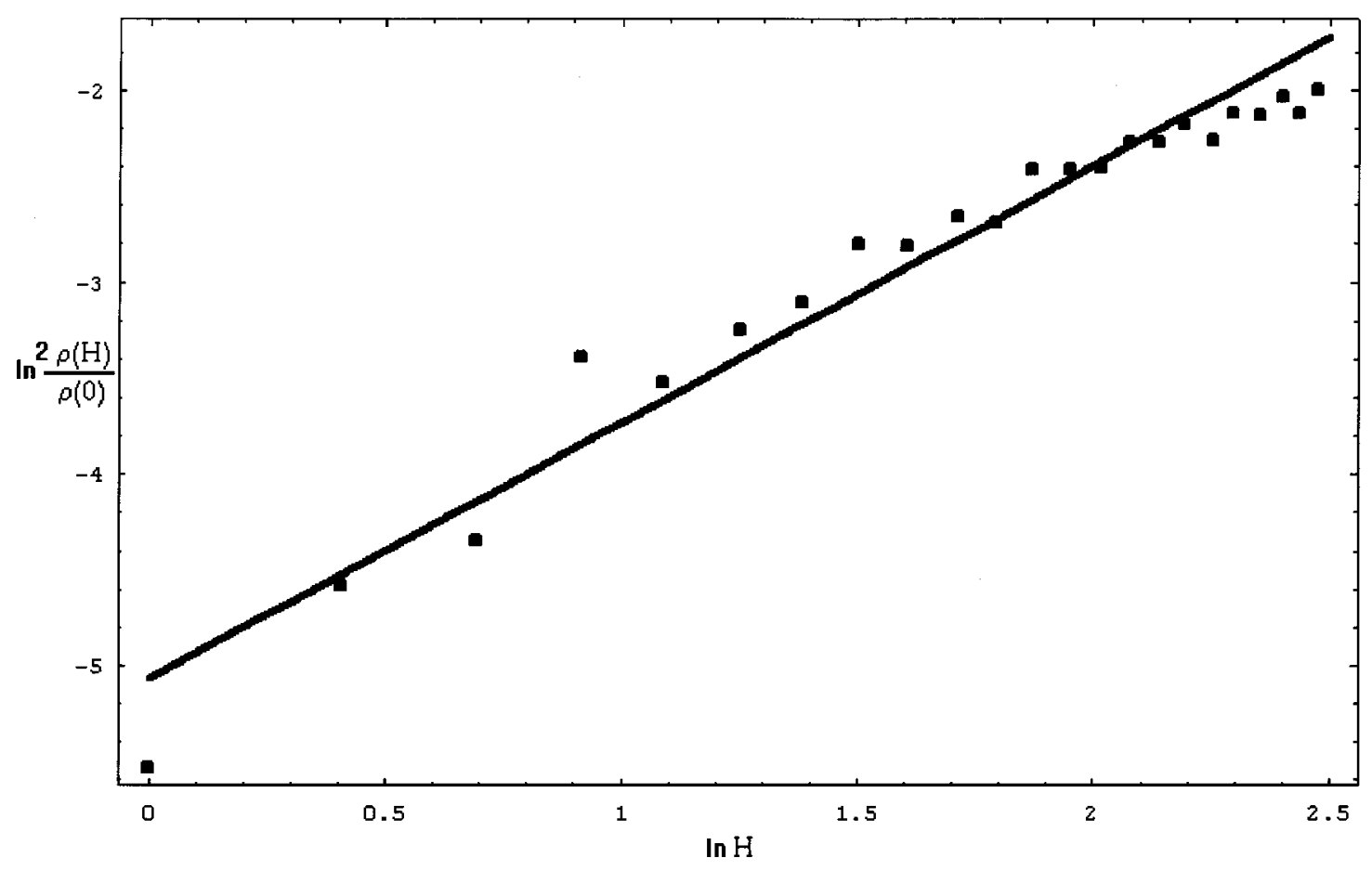

FIG. 1. Magnetoresistance at $4.2 \mathrm{~K}$ from van Ancum et al. (Ref. 5) with theoretical fit.

magnetic-field behavior depends on the form of the scattering length given in Eqs. (4.2), (4.3), and (4.4). For example, for $\lambda \ll\left(\alpha N^{1 / 2}\right)^{-1 / 2}$ we find an $H^{1 / 3}$ dependency for the constant DOS and $H^{1 / 2}$ for electron-electron interactions. For zero electric fields the expression for $\sigma_{e}$ reduces to

$$
\sigma_{e}=\sigma_{0} \exp \left[-\left(\frac{T}{T_{e}}\right)^{-1 / 2}\right]
$$

with $T_{e} \propto H$. The above expression is in agreement with Shklovskii's two-dimensional percolation magnetoconductance result. ${ }^{6}$

It is interesting to note that the temperature dependences of the conductivities in the presence and absence of scattering given by Eqs. (4.6) and (3.6) respectively, are quite different. Therefore, one can observe the effect of scattering in QTD materials by measuring the temperature dependence of the magnetoconductivity. Similar conclusions were drawn for three-dimensional materials. ${ }^{6}$ The electric-field dependences of the QTD magnetoconductance in the presence [Eq. (4.6)] and absence [Eqs. (3.6), (3.3)] of scattering are also different. Hence, one can also observe the effect of scattering through the electric-field dependence of the magnetoconductivity of a QTD material.

\section{DISCUSSION OF RESULTS}

Recently, van Ancum et al..$^{5}$ have measured the magnetoresistance of PBCO thin films and found an exponential positive magnetoresistance. To explain their experiments, they modified three-dimensional percolation expressions of the magnetoconductance ${ }^{6}$ for the two-dimensional case. They found that the logarithm of the conductivity is proportional to $H^{1 / 2}$ for strong (i.e., $\lambda \ll a_{0}$ ) magnetic fields, and $H^{2}$ for weak (i.e., $\lambda \gg a_{0}$ ) magnetic fields. Their expressions were incomplete in the exponents as they included no mate- rial parameter dependences. This allowed them great latitude in fitting the experimental data by using these expressions.

We tried to explain the above experimental results by using our theoretical expressions. Their experiments were performed at very low electric fields, therefore we have neglected the effect of electric fields in our calculations. The experiments were also performed at very low temperatures where electron-electron interactions have been shown to be important in $\mathrm{PBCO}{ }^{3}$ We used the magnetoconductance expressions derived for electron-electron interactions to explain their data. Note that our magnetoconductivity expressions include complete exponential and pre-exponential parts. In the absence of scattering, we did not find a good agreement between theory and experiment. This contradicts the finding of van Ancum et al. ${ }^{5}$ When we included the effect of scattering in our calculation [Eq. (4.6) for electron-electron interactions] a good agreement between theory and experiment was obtained. The best fit was found when we used Eq. (4.3) for the scattering length. Equation (4.3) can be rewritten as

$$
b=\frac{1}{\alpha}\left[1-\kappa H^{4 / 3}\right], \quad\left(\alpha N^{1 / 2}\right)^{-1 / 2} \ll \lambda \ll N^{-1 / 2},
$$

where $\kappa=q^{\prime}\left(e / N^{1 / 2} \alpha \hbar\right)^{4 / 3}$. To fit the above experiments we used $\kappa$ as the only fitting parameter. A good agreement was found for $\kappa=7 \times 10^{-4} \mathrm{~T}^{-4 / 3}$. The values of the other parameters were taken from Ref. 4 as $D_{1}=7.4 \times 10^{56} 1 / \mathrm{J}^{2} \mathrm{~m}^{2}$ and $1 / \alpha=8 \times 10^{-9} \mathrm{~m}$. The theoretical results along with the experiment data are presented in Fig. 1. One can see that there are slight discrepancies between theory and experiment below $\ln H \sim 0.3$ and above $\ln H \sim 2.3$. The disagreement may be due to the simplicity of the wave function [Eq. (4.1)] and the approximations made to derive the analytical result of the magnetoconductivity. Using the value of $b$ given by Eq. (5.1), we found that the logarithm of the conductivity is pro- 


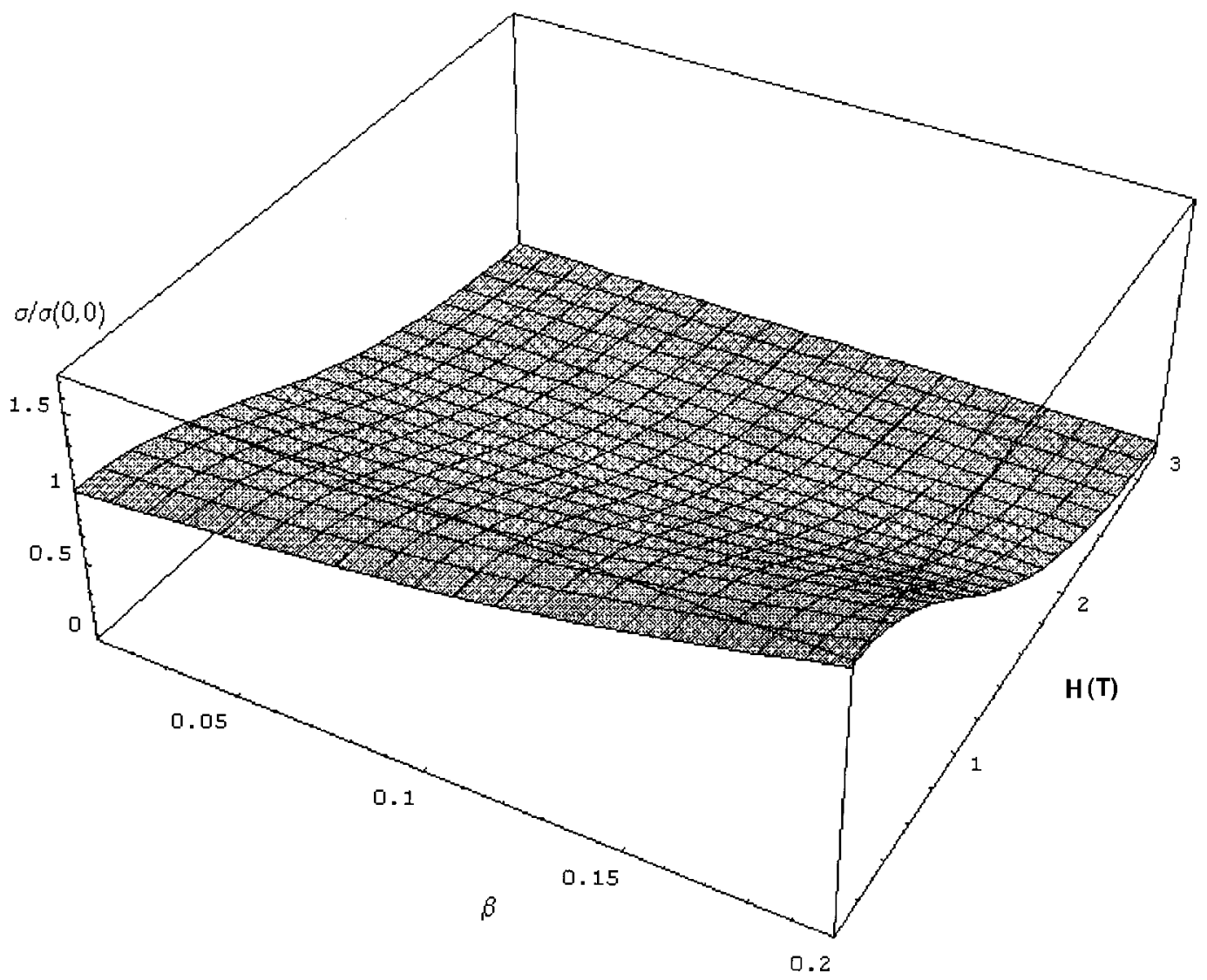

FIG. 2. Predictions for weak magnetoconductance in PBCO at various electric fields for nonscattering case.

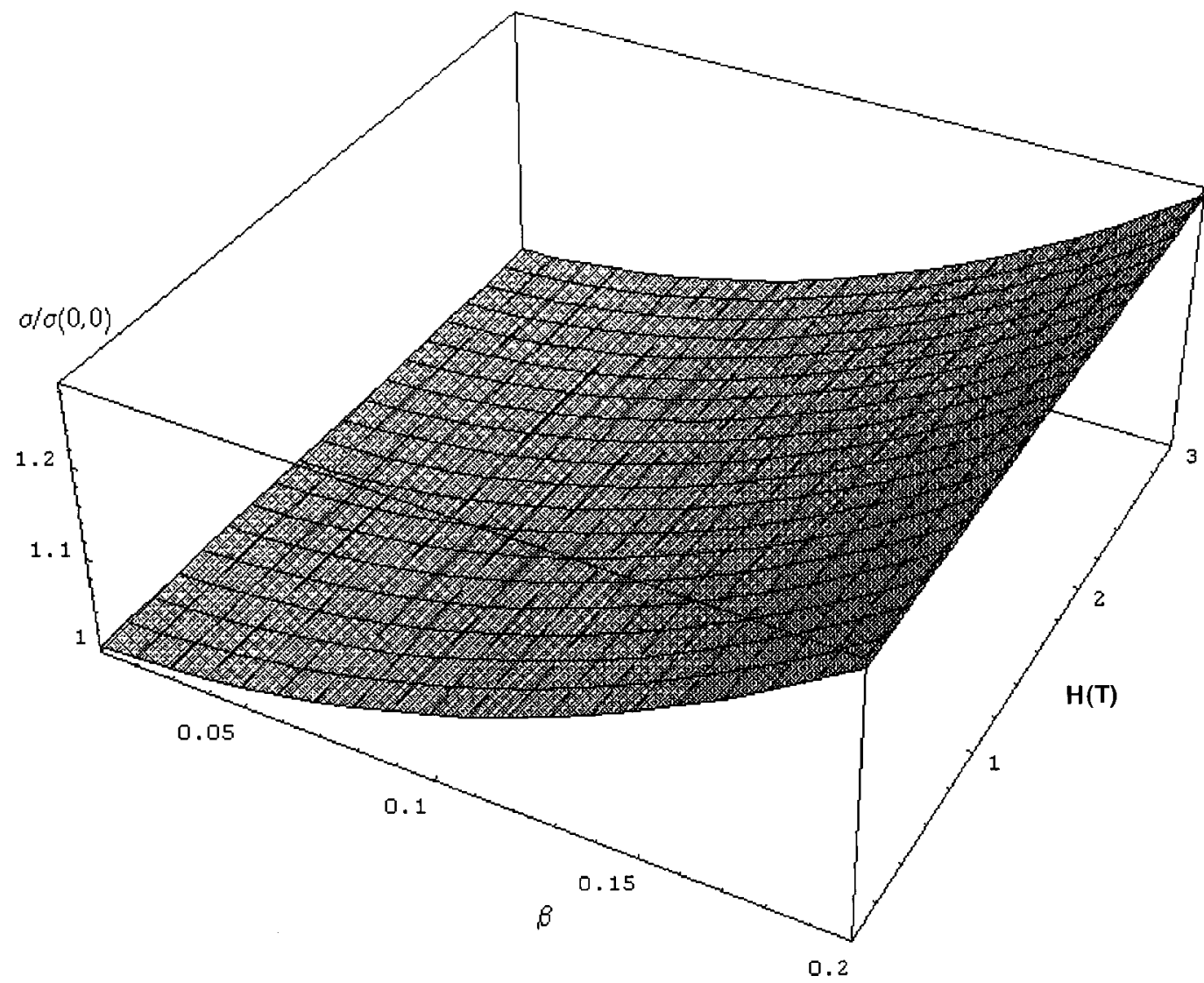

FIG. 3. Predictions for weak magnetoconductance in PBCO at various electric fields for scattering case. 
portional to $\left[1 / \alpha\left(1-\kappa H^{4 / 3}\right)\right]^{-1 / 2}$. Since $\kappa$ is small, we can expand the given expression in a Taylor series. Finally, we get that the logarithm of the conductivity varies as $H^{4 / 3}$ for the PBCO thin film.

From the condition $\left(\alpha N^{1 / 2}\right)^{-1 / 2} \ll \lambda \ll N^{-1 / 2}$ given in Eq. (5.1), we can put bounds on the value of the two-dimensional concentration $N$. From this condition, we have estimated that the value of $N$ lies between $10^{11}$ and $10^{12} \mathrm{~cm}^{-2}$. This in turn allows us to estimate the maximum value of the unknown parameter $q^{\prime} \sim 10^{-2}$ which appears in the expression of $\kappa$. Kabasawa et al. ${ }^{1}$ proposed a condition for metal-to-insulator transition in PBCO which is expressed as $N^{1 / 2} / \alpha \sim 0.85$. This equation gives an $N$ of about $10^{12} \mathrm{~cm}^{-2}$. Hence, one can conclude that the PBCO samples of van Ancum et al. are near the criterion of metal-insulator transition.

Using the parameters obtained from Fig. 1, we have also calculated the conductivity in the presence and absence of scattering as a function of electric and magnetic fields for the PBCO thin films. The electron-electron interaction magnetoconductance formulas are used to calculate Figs. 2 and 3 for the nonscattering [Eq. (3.3)] and scattering [Eq. (4.6)] cases, respectively. We chose a range of magnetic field strength which falls within the range of the van Ancum experiment. By comparing these two figures, one can see clearly the effect of scattering on the electric and magnetic-field dependence of the magnetoconductance.

\section{ACKNOWLEDGMENTS}

M.S. is thankful to NSERC of Canada for financial support. R.B.T. would like to thank OGS for financial support.

\section{APPENDIX}

The parameters appearing in Eq. (3.3) are given below.

$$
\begin{gathered}
c_{1}^{c}=\frac{1}{2}+\frac{2 \beta}{\pi}+\eta_{1}, \quad c_{2}^{c}=\frac{1}{2}+\eta_{1}, \quad c_{3}^{c}=\frac{1}{2}+\frac{5 \beta}{\pi}+\eta_{4}, \\
c_{4}^{c}=\frac{1}{2}+\eta_{4}, \quad c_{1}^{e}=\frac{1}{2}+\frac{2 \beta}{\pi}+\frac{3 \beta^{2}}{4}+\eta_{1}, \\
c_{2}^{e}=c_{1}^{c}, \quad c_{3}^{e}=\frac{1}{2}+\frac{5 \beta}{\pi}+\frac{15 \beta^{2}}{4}+\eta_{4}, \quad c_{4}^{e}=c_{3}^{c} . \\
c_{5}^{c}=\frac{\pi \eta_{3}}{3}-\frac{\pi \beta}{6}-\frac{1}{3}, \quad c_{6}^{c}=\frac{\pi \eta_{6}}{5}-\frac{\pi \beta}{4}-\frac{1}{5}, \\
c_{7}^{c}=\frac{\pi \eta_{2}}{2}+\frac{\pi}{4}+\frac{\beta}{2}, \quad c_{8}^{c}=\frac{\pi \eta_{5}}{4}+\frac{\pi}{8}+\beta, \\
c_{5}^{e}=\frac{\pi}{2}-\frac{1}{2}-\frac{\pi \beta}{4}-\beta^{2}, \quad c_{6}^{e}=\frac{\pi \eta_{6}}{5}-\frac{1}{5}-\frac{\pi \beta}{4}-2 \beta^{2}, \\
c_{9}^{c}=\left(c_{1}^{c}\right)^{-1 / 3}, \quad c_{10}^{c}=\frac{2}{5}\left(\frac{T_{c}}{T}\right) \frac{c_{3}^{c}}{\left(c_{1}^{c}\right)^{2}},
\end{gathered}
$$

$$
\begin{gathered}
c_{7}^{e}=\pi \eta_{2}+\frac{\pi}{2}+\beta+\frac{\pi \beta^{2}}{4}, \\
c_{8}^{e}=\frac{3 \pi \eta_{5}}{10}+\frac{3 \pi}{20}+\frac{6 \beta}{5}+\frac{3 \pi \beta^{2}}{4}, \quad c_{9}^{e}=\left(\frac{T_{e}}{T}\right)^{1 / 2}\left(c_{1}^{e}\right)^{-1 / 4}, \\
c_{10}^{e}=\frac{1}{5}\left(\frac{T_{e}}{T}\right)^{3 / 2} \frac{c_{3}^{e}}{\left(c_{1}^{e}\right)^{7 / 4}} .
\end{gathered}
$$

The factors $\eta_{1}$ through $\eta_{6}$ are functions of $\beta . \eta_{1}, \eta_{2}$, and $\eta_{3}$ can be found in our previous work, ${ }^{4}$ and $\eta_{4}, \eta_{5}$, and $\eta_{6}$ are given as

$$
\begin{gathered}
\eta_{4}=\frac{83}{315 \pi} \quad \beta=1, \\
\eta_{4}=\frac{\beta\left(6 \beta^{6}-23 \beta^{4}+8 \beta^{2}-96\right)}{24 \pi\left(1-\beta^{2}\right)^{4}}+\frac{\left(8+24 \beta^{2}+3 \beta^{4}\right)}{4 \pi\left(1-\beta^{2}\right)^{9 / 2}} \\
\quad \times \tan ^{-1}\left(\frac{1-\beta}{\sqrt{1-\beta^{2}}}\right) \quad \beta<1, \\
\eta_{4}=\frac{\beta\left(6 \beta^{6}-23 \beta^{4}+8 \beta^{2}-96\right)}{24 \pi\left(\beta^{2}-1\right)^{4}}+\frac{\left(8+24 \beta^{2}+3 \beta^{4}\right)}{8 \pi\left(\beta^{2}-1\right)^{9 / 2}} \\
\quad \times \cosh ^{-1}(\beta) \quad \beta>1 . \\
\eta_{5}=\frac{12}{35 \pi} \quad \beta=1,
\end{gathered}
$$$$
\eta_{5}=\frac{\beta\left(5 \beta^{2}-2 \beta^{4}-18\right)}{6 \pi\left(1-\beta^{2}\right)^{3}}+\frac{\left(2+3 \beta^{2}\right)}{\pi\left(1-\beta^{2}\right)^{7 / 2}}
$$$$
\times \tan ^{-1}\left(\frac{1-\beta}{\sqrt{1-\beta^{2}}}\right) \quad \beta<1,
$$$$
\eta_{5}=\frac{-\beta\left(5 \beta^{2}-2 \beta^{4}-18\right)}{6 \pi\left(\beta^{2}-1\right)^{3}}-\frac{\left(2+3 \beta^{2}\right)}{2 \pi\left(\beta^{2}-1\right)^{7 / 2}}
$$$$
\times \cosh ^{-1}(\beta) \quad \beta>1
$$$$
\eta_{6}=\frac{5}{63 \pi} \quad \beta=1
$$$$
\eta_{6}=\frac{\left(2 \beta^{6}-5 \beta^{4}+84 \beta^{2}+24\right)}{24 \pi\left(1-\beta^{2}\right)^{4}}-\frac{5 \beta\left(4+3 \beta^{2}\right)}{4 \pi\left(1-\beta^{2}\right)^{9 / 2}}
$$$$
\times \tan ^{-1}\left(\frac{1-\beta}{\sqrt{1-\beta^{2}}}\right) \quad \beta<1 \text {, }
$$$$
\eta_{6}=\frac{\left(2 \beta^{6}-5 \beta^{4}+84 \beta^{2}+24\right)}{24 \pi\left(\beta^{2}-1\right)^{4}}-\frac{5 \beta\left(4+3 \beta^{2}\right)}{8 \pi\left(\beta^{2}-1\right)^{9 / 2}}
$$$$
\times \cosh ^{-1}(\beta) \quad \beta>1 \text {. }
$$ 
${ }^{1}$ U. Kabasawa et al., Phys. Rev. Lett. 70, 1700 (1993); U. Kabasawa et al., Physica C 194, 261 (1992).

${ }^{2}$ D. Seong et al., Solid State Commun. 76, 1341 (1990); Yu. M. Boguslavskij et al., Physica B 194, 1115 (1994); M. Koyanagi et al., ibid. 194, 2155 (1994).

${ }^{3}$ M. Singh et al., Phys. Rev. B 50, 7007 (1994); 53, 6806 (1996); Phys. Status Solidi B 197, 65 (1996).

${ }^{4}$ R. B. Thompson and M. Singh, Philos. Mag. B 75, 293 (1997).

${ }^{5}$ G. K. van Ancum et al., Phys. Rev. B 52, 15644 (1995).

${ }^{6}$ B. I. Shklovskii and A. L. Efros, Electronic Properties of Doped Semiconductors (Springer-Verlag, New York, 1984); B. I. Shklovskii, JETP Lett. 36, 51 (1982); Sov. Phys. Semicond. 17, 1311 (1983).
${ }^{7}$ A. Hartstein, A. B. Fowler, and K. C. Woo, Physica B 117-118, Part II 655 (1983).

${ }^{8}$ H. Tokumoto et al., Solid State Commun. 35, 961 (1980); Philos. Mag. 46, 93 (1982).

${ }^{9}$ M. Pollak, The Metal Non-Metal Transition in Disordered Systems, edited by L. R. Friedman and D. P. Tunstall (SUSSP Publications, Edinburgh, 1978).

${ }^{10}$ N. F. Mott and E. A. Davis, Electron Processes in NonCrystalline Materials (Oxford, London, 1971).

${ }^{11}$ N. Apsley and H. P. Hughes, Philos. Mag. 30, 963 (1974); 31, 1327 (1975).

${ }^{12}$ B. I. Shklovskii and A. L. Efros, Sov. Phys. JETP 57, 470 (1983). 